\title{
Inverse Nonnegativity of Tridiagonal $M$-Matrices under Diagonal Element-Wise Perturbation
}

\author{
Mohamed A. Ramadan', Mahmoud M. Abu Murad² \\ ${ }^{1}$ Department of Mathematics, Faculty of Science, Menoufia University, Shebeen El-Kom, Egypt \\ ${ }^{2}$ Department of Mathematics, Faculty of Science, Zagazig University, Ash Sharqiyah, Egypt \\ Email: ramadanmohamed13@yahoo.com, mramadan@eun.eg, abomorad1978@yahoo.com, \\ mabumurad@su.edu.sa
}

Received 24 March 2015; accepted 6 June 2015; published 9 June 2015

Copyright (C) 2015 by authors and Scientific Research Publishing Inc.

This work is licensed under the Creative Commons Attribution International License (CC BY). http://creativecommons.org/licenses/by/4.0/

\section{(c) (i) Open Access}

\begin{abstract}
One of the most important properties of $M$-matrices is element-wise non-negative of its inverse. In this paper, we consider element-wise perturbations of tridiagonal $M$-matrices and obtain bounds on the perturbations so that the non-negative inverse persists. The largest interval is given by which the diagonal entries of the inverse of tridiagonal $M$-matrices can be perturbed without losing the property of total nonnegativity. A numerical example is given to illustrate our findings.
\end{abstract}

\section{Keywords}

Totally Positive Matrix, Totally Nonnegative Matrix, Tridiagonal Matrices, Compound Matrix, Element-Wise Perturbations

\section{Introduction}

In many mathematical problems, $Z$-matrices and $M$-matrices play an important role. It is often useful to know the properties of their inverses, especially when the $Z$-matrices and the $M$-matrices have a special combinatorial structure, for more details we refer the reader [1]. $M$-matrices have important applications, for instance, in iterative methods, in numerical analysis, in the analysis of dynamical systems, in economics, and in mathematical programming. One of the most important properties of some kinds of $M$-matrices is the nonegativity of their inverses, which plays central role in many of mathematical problems.

An $n \times n$ real matrix $M=\left(m_{i j}\right)$ is called $M$-matrix if $m_{i i}>0, i=1, \cdots, n$ and $m_{i j} \leq 0, i \neq j$, over the 
years, $M$-matrices have considerable attention, in large part because they arise in many applications [2] [3]. Recently, a noticeable amount of attention has turned to the inverse of tridiagonal $M$-matrices (those matrices which happen to be inverses of special form of $M$-matrices with property $a_{i j}=0$ whenever $|i-j|>1$ ) and $M$ is generalized strictly diagonally dominant. A matrix is said to be generalized (strictly) diagonally dominant

if $m_{i i}>\sum_{\substack{i=1 \\ i \neq j}}^{n} m_{i j}$. Of particular importance to us is the fact that since $M$ is an $M$-matrix it is non-singular and

$M^{-1}>0$ where the inequality is satisfied element-wise. A rich class of $M$-matrices were introduced by Ostrowski in 1937 [4], with reference to the work of Minkowski [5] [6]. A condition which is easy to check is that a matrix $M$ is an $M$-matrix if and only if $m_{i j} \leq 0, i \neq j$ and $m_{i i}>0$, and $M$ is generalized strictly diagonally dominant.

In this paper, we consider the inverse of perturbed $M$-matrix. Specifically we consider the effect of changing single elements inside the diagonal of $M^{-1}$. We are interested in the large amount by which the single diagonal element of $M^{-1}$ can be varied without losing the property of total nonnegativity.

The reminder of the paper is organized as follows. In section 2, we explain our notations and some needed important definitions are presented. In section 3, some auxiliary results and important prepositions and lemmas are stated. In section 4, we present our results.

\section{Notations}

In this section we introduce the notation that will be used in developing the paper. For $k, n$ we denote by $Q_{k, n}$ the set of all strictly increasing sequences of $k$ integers chosen from $\{1,2, \cdots, n\}$. For $\alpha=\left\{\alpha_{1}, \cdots, \alpha_{k}\right\}$, $\beta=\left\{\beta_{1}, \cdots, \beta_{k}\right\} \in Q_{k, n}$, we denote by $A[\alpha \mid \beta]$ the $k \times k$ submatrix of $A$ contained in the rows indexed by $\alpha_{1}, \cdots, \alpha_{k}$ and columns indexed by $\beta_{1}, \cdots, \beta_{k}$. A matrix $A$ is called totally positive (abbreviated TP henceforth) if $\operatorname{det} A[\alpha \mid \beta]>0$ and totally nonnegative (abbreviated TN) if $\operatorname{det} A[\alpha \mid \beta] \geq 0$ for all $\alpha, \beta \in Q_{k, n}$, $k=1,2, \cdots, n$. For a given index $\alpha=\left\{\alpha_{1}, \cdots, \alpha_{k}\right\}$, with property $\alpha_{j}<\alpha_{j+1}, j=1, \cdots, k-1$, the dispersion of $\alpha$, denoted by $d(\alpha)$, is defined to be $d(\alpha)=\sum_{j=1}^{k-1} \alpha_{j+1}-\alpha_{j}-1=\alpha_{k}-\alpha_{1}-k+1$.

Throughout this paper we use the following notation for general tridiagonal $M$-matrix:

$$
M=\left[\begin{array}{ccccc}
a_{1} & -b_{1} & 0 & \cdots & 0 \\
-c_{1} & a_{2} & -b_{2} & & \vdots \\
0 & \ddots & \ddots & \ddots & 0 \\
\vdots & & -c_{n-2} & a_{n-1} & -b_{n-1} \\
0 & \cdots & 0 & -c_{n-1} & a_{n}
\end{array}\right]
$$

where $a_{i}, b_{i}$ and $c_{i}>0$, and each $a_{i}$ is large enough that $M$ is strictly diagonally dominant.

We let $E_{i j}$ to be the square standard basis matrix whose only nonzero entry is 1 that occurs in the $(i, j)$ position.

Definition 2.1 Compound Matrices ([7], p. 19).

Let $A=\left(a_{i j}\right)_{i, j=1}^{n}$ be a square matrix of order $n$. Let $\alpha=\{1, \cdots, n\}$ be the index set of cardinality $n$, defining $\alpha_{i}=\alpha \backslash\{n-i+1\}, \quad i=\{1, \cdots, n\}$ are the index sets of cardinality $n-1$.

Construct the following table which depends on $\alpha_{i}$.

\begin{tabular}{ccccc}
\hline & $\alpha_{1}$ & $\alpha_{2}$ & $\ldots$ & $\alpha_{n}$ \\
\hline$\alpha_{1}$ & $\operatorname{det} A\left(\alpha_{1}, \alpha_{1}\right)$ & $\operatorname{det} A\left(\alpha_{1}, \alpha_{2}\right)$ & $\ldots$ & $\operatorname{det} A\left(\alpha_{1}, \alpha_{n}\right)$ \\
$\alpha_{2}$ & $\operatorname{det} A\left(\alpha_{2}, \alpha_{1}\right)$ & $\operatorname{det} A\left(\alpha_{2}, \alpha_{2}\right)$ & $\ldots$ & $\operatorname{det} A\left(\alpha_{2}, \alpha_{n}\right)$ \\
$\vdots$ & $\vdots$ & $\vdots$ & $\ldots$ & $\vdots$ \\
$\alpha_{n}$ & $\operatorname{det} A\left(\alpha_{n}, \alpha_{1}\right)$ & $\operatorname{det} A\left(\alpha_{n}, \alpha_{2}\right)$ & $\ldots$ & $\operatorname{det} A\left(\alpha_{n}, \alpha_{n}\right)$ \\
\hline
\end{tabular}


The created matrix $C_{n-1}(A)=\left[\begin{array}{cccc}\operatorname{det} A\left(\alpha_{1}, \alpha_{1}\right) & \operatorname{det} A\left(\alpha_{1}, \alpha_{2}\right) & \cdots & \operatorname{det} A\left(\alpha_{1}, \alpha_{n}\right) \\ \operatorname{det} A\left(\alpha_{2}, \alpha_{1}\right) & \operatorname{det} A\left(\alpha_{2}, \alpha_{2}\right) & \cdots & \operatorname{det} A\left(\alpha_{2}, \alpha_{n}\right) \\ \vdots & \vdots & \cdots & \vdots \\ \operatorname{det} A\left(\alpha_{n}, \alpha_{1}\right) & \operatorname{det} A\left(\alpha_{n}, \alpha_{2}\right) & \cdots & \operatorname{det} A\left(\alpha_{n}, \alpha_{n}\right)\end{array}\right]$

is called $(n-1)^{\text {th }}$, compound matrix of $A$.

For example, if

$A=\left[\begin{array}{lll}1 & 2 & 3 \\ 4 & 5 & 6 \\ 7 & 8 & 9\end{array}\right]$ with indexed sets $\alpha_{1}=\{1,2\}, \alpha_{2}=\{1,3\}$ and $\alpha_{3}=\{2,3\}$.

Then $C_{2}(A)=\left[\begin{array}{lll}\operatorname{det} A\left(\alpha_{1}, \alpha_{1}\right) & \operatorname{det} A\left(\alpha_{1}, \alpha_{2}\right) & \operatorname{det} A\left(\alpha_{1}, \alpha_{3}\right) \\ \operatorname{det} A\left(\alpha_{2}, \alpha_{1}\right) & \operatorname{det} A\left(\alpha_{2}, \alpha_{2}\right) & \operatorname{det} A\left(\alpha_{2}, \alpha_{3}\right) \\ \operatorname{det} A\left(\alpha_{3}, \alpha_{1}\right) & \operatorname{det} A\left(\alpha_{3}, \alpha_{2}\right) & \operatorname{det} A\left(\alpha_{3}, \alpha_{3}\right)\end{array}\right]=\left[\begin{array}{ccc}-3 & -6 & -3 \\ -6 & -12 & -6 \\ -3 & -6 & -3\end{array}\right]$.

\section{Auxiliary Results}

We start with some basic facts on tridiagonal $M$-matrices. We can find the determinant of any $n \times n$ tridiagonal $M$-matrix $\quad M=\left(m_{i j}\right)$ by using the following recursion equation [8] [9].

$$
\begin{aligned}
\operatorname{det} M & =a_{1} \operatorname{det} M(2, \cdots, n)-b_{1} c_{1} \operatorname{det} M(3, \cdots, n) \\
& =a_{n} \operatorname{det} M(1, \cdots, n-1)-b_{n-1} c_{n-1} \operatorname{det} M(1, \cdots, n-2) .
\end{aligned}
$$

And we have the following proposition for finding the determinant of a $n \times n$ tridiagonal $M$-matrix $M=\left(m_{i j}\right)$.

Proposition 3.1 ([10], formula 4.1) For any $n \times n$ tridiagonal $M$-matrix $M=\left(m_{i j}\right)$ the following relation is true

$$
\operatorname{det} M=\operatorname{det} M(1, \cdots, i-1) \operatorname{det} M(i+1, \cdots, n)-b_{i} c_{i} \operatorname{det} M(1, \cdots, i-2) \operatorname{det} M(i+1, \cdots, n), \quad i=2, \cdots, n .
$$

We will present now some of propositions of nonsingular totally nonnegative matrices which important for our work.

Proposition 3.2 [10] [11]

For any nonsingular totally nonnegative matrix $A=\left(a_{i j}\right)_{i, j=1}^{n}$, all principle minors are positive.

That is $\operatorname{det} A(\alpha)>0$, for all $k$ and $\alpha \in Q_{n, k}$.

Proposition 3.3 ([7], p. 21)

Let $M$ be a nonsingular tridiagonal $M$-matrix, and $M^{-1}$ be the inverse of the matrix $M$ then

$$
\operatorname{det} M^{-1}=\frac{\operatorname{det} M^{-1}(\alpha)}{\operatorname{det} M\left(\alpha^{\prime}\right)} \text {, when } d(\alpha)=0 .
$$

In the sequel we will make use the following lemma, see, e.g. [12].

Lemma 3.4 (Sylvester Identity)

Partition square matrix $p$ of order $n, n>2$, as:

$$
P=\left[\begin{array}{ccc}
a & P_{12} & b \\
P_{21} & P_{22} & P_{23} \\
c & P_{32} & d
\end{array}\right],
$$

where $P_{22}$ square matrix of order $n-2$ and $a, b, c$ and $d$ are scalars.

Define the submatrices

$$
\begin{aligned}
& A=\left[\begin{array}{cc}
a & P_{12} \\
P_{21} & P_{22}
\end{array}\right], B=\left[\begin{array}{cc}
P_{12} & b \\
P_{22} & P_{23}
\end{array}\right] \\
& C=\left[\begin{array}{ll}
P_{21} & P_{22} \\
c & P_{32}
\end{array}\right], D=\left[\begin{array}{cc}
P_{22} & P_{23} \\
P_{32} & d
\end{array}\right]
\end{aligned}
$$


If $P_{22}$ is nonsingular, then

$$
\operatorname{det} P=\frac{\operatorname{det} A \operatorname{det} D-\operatorname{det} B \operatorname{det} C}{\operatorname{det} P_{22}}
$$

Lemma 3.5 ([11], p.199) Let $A=\left(a_{i j}\right)$ be a square matrix of order $n$, with $\operatorname{det} A(2, \cdots, n) \neq 0$. Then $A-x E_{11}$ is totally nonnegative $\forall x \in\left[0, \frac{\operatorname{det} A}{\operatorname{det} A(2, \cdots, n)}\right]$.

We now state an important result which links the determinant of $M$-matrix with the value of the elements of its inverse.

Lemma 3.6 [10] Let $M=\left[m_{i j}\right]_{i, j=1}^{n}$ be a tridiagonal matrix of order $n$, then we can find the elements of inverse matrix $M^{-1}=\left[m_{i j}^{-1}\right]_{i, j=1}^{n}$ by using the following formula

$$
m_{i j}^{-1}=(-1)^{i+j} \frac{\operatorname{det} M(1,2, \cdots, \hat{j}, \cdots, n / 1,2, \cdots, \hat{i}, \cdots, n)}{\operatorname{det} M} .
$$

\section{Main Results}

In this section, we present our results based on the inverse of tridiagonal $M$-matrices. Firstly we begin with the following theorem.

Theorem 4.1

Let $M=\left[\begin{array}{ccccc}a_{1} & -b_{1} & 0 & \cdots & 0 \\ -c_{1} & a_{2} & -b_{2} & & \vdots \\ 0 & \ddots & \ddots & \ddots & 0 \\ \vdots & & -c_{n-2} & a_{n-1} & -b_{n-1} \\ 0 & \cdots & 0 & -c_{n-1} & a_{n}\end{array}\right]$ be strictly diagonally dominant $M$-matrix.

If $C_{n-1}(M)$ is the $(n-1)^{\text {th }}$ compound matrix of $M$ then the matrix $S\left(C_{n-1}(M)\right)^{\mathrm{T}} S$ is totally nonnegative matrix. Moreover, $\operatorname{det} S\left(C_{n-1}(M)\right)^{\mathrm{T}} S=(\operatorname{det} M)^{n-1}$ where $S=\operatorname{diag}\left(1,-1, \cdots,(-1)^{n+1}\right)$

Proof: Let $M$ be strictly diagonally dominant $M$-matrix.

Then $M^{-1}=\frac{S\left(C_{n-1}(M)\right)^{\mathrm{T}} S}{\operatorname{det} M}$ is totally nonnegative matrix. So is $S\left(C_{n-1}(M)\right)^{\mathrm{T}} S$.

You can find this formula in ([7], p. 21).

There is an explicit formula for the determinant of $\operatorname{det} S\left(C_{n-1}(M)\right)^{\mathrm{T}} S=(\operatorname{det} M)^{n-1}$ given as

$$
\begin{aligned}
& \operatorname{det} S\left(C_{n-1}(M)\right)^{\mathrm{T}} S=\left|\begin{array}{cccc}
\operatorname{det} M\left(\alpha_{1}, \alpha_{1}\right) & -\operatorname{det} M\left(\alpha_{2}, \alpha_{1}\right) & \cdots & (-1)^{n+1} \operatorname{det} M\left(\alpha_{n}, \alpha_{1}\right) \\
-\operatorname{det} M\left(\alpha_{1}, \alpha_{2}\right) & \operatorname{det} M\left(\alpha_{2}, \alpha_{2}\right) & \cdots & (-1)^{n+2} \operatorname{det} M\left(\alpha_{n}, \alpha_{2}\right) \\
\vdots & \vdots & \cdots & \vdots \\
(-1)^{n+1} \operatorname{det} M\left(\alpha_{1}, \alpha_{2}\right) & (-1)^{n+2} \operatorname{det} M\left(\alpha_{2}, \alpha_{n}\right) & \cdots & (-1)^{2 n} \operatorname{det} M\left(\alpha_{n}, \alpha_{n}\right)
\end{array}\right| \\
& =\operatorname{det} A\left(\alpha_{1}, \alpha_{1}\right)\left|\begin{array}{cccc}
1 & -\frac{\operatorname{det} M\left(\alpha_{2}, \alpha_{1}\right)}{\operatorname{det} M\left(\alpha_{1}, \alpha_{1}\right)} & \cdots & (-1)^{n+1} \frac{\operatorname{det} M\left(\alpha_{n}, \alpha_{1}\right)}{\operatorname{det} M\left(\alpha_{1}, \alpha_{1}\right)} \\
-\operatorname{det} M\left(\alpha_{1}, \alpha_{2}\right) & \operatorname{det} M\left(\alpha_{2}, \alpha_{2}\right) & \cdots & (-1)^{n+2} \operatorname{det} M\left(\alpha_{n}, \alpha_{2}\right) \\
\vdots & \vdots & \cdots & \vdots \\
(-1)^{n+1} \operatorname{det} M\left(\alpha_{1}, \alpha_{n}\right) & (-1)^{n+2} \operatorname{det} M\left(\alpha_{2}, \alpha_{n}\right) & \cdots & \operatorname{det} M\left(\alpha_{n}, \alpha_{n}\right)
\end{array}\right| .
\end{aligned}
$$


Multiply the first row by $(-1)^{i} \operatorname{det} A\left(\alpha_{1}, \alpha_{i}\right)$ and add it to the $i^{\text {th }}$ row to obtain

$$
\begin{aligned}
& =\operatorname{det} M\left(\alpha_{1}, \alpha_{1}\right)\left|\begin{array}{cccc}
1 & -\frac{\operatorname{det} M\left(\alpha_{2}, \alpha_{1}\right)}{\operatorname{det} M\left(\alpha_{1}, \alpha_{1}\right)} & \ldots & (-1)^{n+1} \frac{\operatorname{det} M\left(\alpha_{n}, \alpha_{1}\right)}{\operatorname{det} M\left(\alpha_{1}, \alpha_{1}\right)} \\
0 & \frac{\operatorname{det} M\left(\alpha_{2}, \alpha_{2}\right) \operatorname{det} M\left(\alpha_{1}, \alpha_{1}\right)-\operatorname{det} M\left(\alpha_{2}, \alpha_{1}\right) \operatorname{det} M\left(\alpha_{1}, \alpha_{2}\right)}{\operatorname{det} M\left(\alpha_{1}, \alpha_{1}\right)} & \cdots & \frac{(-1)^{n+2} \operatorname{det} M\left(\alpha_{n}, \alpha_{2}\right) \operatorname{det} M\left(\alpha_{1}, \alpha_{1}\right)+(-1)^{n+1} \operatorname{det} M\left(\alpha_{n}, \alpha_{1}\right) \operatorname{det} M\left(\alpha_{1}, \alpha_{2}\right)}{\operatorname{det} A\left(\alpha_{1}, \alpha_{1}\right)} \\
\vdots & \vdots & \ldots & \vdots \\
0 & \frac{(-1)^{n+2} \operatorname{det} M\left(\alpha_{2}, \alpha_{n}\right) \operatorname{det} M\left(\alpha_{1}, \alpha_{1}\right)+(-1)^{n+1} \operatorname{det} M\left(\alpha_{1}, \alpha_{n}\right) \operatorname{det} M\left(\alpha_{2}, \alpha_{1}\right)}{\operatorname{det} M\left(\alpha_{1}, \alpha_{1}\right)} & \cdots & \frac{\operatorname{det} M\left(\alpha_{n}, \alpha_{n}\right) \operatorname{det} M\left(\alpha_{1}, \alpha_{1}\right)+(-1)^{2 n+1} \operatorname{det} M\left(\alpha_{1}, \alpha_{n}\right) \operatorname{det} M\left(\alpha_{n}, \alpha_{1}\right)}{\operatorname{det} M\left(\alpha_{1}, \alpha_{1}\right)}
\end{array}\right| \\
& =\frac{1}{\left(\operatorname{det} M\left(\alpha_{1}, \alpha_{1}\right)\right)^{n-1}}\left|\begin{array}{cccc}
1 & -\frac{\operatorname{det} M\left(\alpha_{2}, \alpha_{1}\right)}{\operatorname{det} M\left(\alpha_{1}, \alpha_{1}\right)} & \cdots & (-1)^{n+1} \frac{\operatorname{det} M\left(\alpha_{n}, \alpha_{1}\right)}{\operatorname{det} M\left(\alpha_{1}, \alpha_{1}\right)} \\
\vdots & \operatorname{det} M\left(\alpha_{2}, \alpha_{2}\right) \operatorname{det} M\left(\alpha_{1}, \alpha_{1}\right)-\operatorname{det} M\left(\alpha_{2}, \alpha_{1}\right) \operatorname{det} M\left(\alpha_{1}, \alpha_{2}\right) & \cdots & (-1)^{n+2} \operatorname{det} M\left(\alpha_{n}, \alpha_{2}\right) \operatorname{det} M\left(\alpha_{1}, \alpha_{1}\right)+(-1)^{n+1} \operatorname{det} M\left(\alpha_{n}, \alpha_{1}\right) \operatorname{det} M\left(\alpha_{1}, \alpha_{2}\right) \\
\vdots & \vdots & \cdots & \vdots \\
0 & (-1)^{n+2} \operatorname{det} M\left(\alpha_{2}, \alpha_{n}\right) \operatorname{det} M\left(\alpha_{1}, \alpha_{1}\right)+(-1)^{n+1} \operatorname{det} M\left(\alpha_{1}, \alpha_{n}\right) \operatorname{det} M\left(\alpha_{2}, \alpha_{1}\right) & \cdots & \operatorname{det} M\left(\alpha_{n}, \alpha_{n}\right) \operatorname{det} M\left(\alpha_{1}, \alpha_{1}\right)+(-1)^{2 n+1} \operatorname{det} M\left(\alpha_{1}, \alpha_{n}\right) \operatorname{det} M\left(\alpha_{n}, \alpha_{1}\right)
\end{array}\right| \\
& =\frac{1}{\left(\operatorname{det} M\left(\alpha_{1}, \alpha_{1}\right)\right)^{n-1}}\left|\begin{array}{cccc}
1 & -\frac{\operatorname{det} M\left(\alpha_{2}, \alpha_{1}\right)}{\operatorname{det} M\left(\alpha_{1}, \alpha_{1}\right)} & \cdots & (-1)^{n+1} \frac{\operatorname{det} M\left(\alpha_{n}, \alpha_{1}\right)}{\operatorname{det} M\left(\alpha_{1}, \alpha_{1}\right)} \\
0 & \operatorname{det} M\left(\left(\alpha_{1}\right)_{1},\left(\alpha_{1}\right)_{1}\right) \operatorname{det} M & \cdots & (-1)^{n} \operatorname{det} M\left(\left(\alpha_{1}\right)_{1},\left(\alpha_{1}\right)_{n-1}\right) \operatorname{det} M \\
\vdots & \vdots & \cdots & \vdots \\
0 & (-1)^{n} \operatorname{det} M\left(\left(\alpha_{1}\right)_{1},\left(\alpha_{1}\right)_{n-1}\right) \operatorname{det} M & \cdots & \operatorname{det} M\left(\left(\alpha_{1}\right)_{n-1},\left(\alpha_{1}\right)_{n-1}\right) \operatorname{det} M
\end{array}\right| . \\
& \text { where }\left(\alpha_{1}\right)_{i}=\alpha_{1} \backslash\{n-i\}=\frac{(\operatorname{det} M)^{n-1}}{\left(\operatorname{det} A\left(\alpha_{1}, \alpha_{1}\right)\right)^{n-1}}\left|\begin{array}{cccc}
1 & -\frac{\operatorname{det} M\left(\alpha_{2}, \alpha_{1}\right)}{\operatorname{det} M\left(\alpha_{1}, \alpha_{1}\right)} & \cdots & (-1)^{n+1} \frac{\operatorname{det} M\left(\alpha_{n}, \alpha_{1}\right)}{\operatorname{det} A\left(\alpha_{1}, \alpha_{1}\right)} \\
0 & \operatorname{det} M\left(\left(\alpha_{1}\right)_{1},\left(\alpha_{1}\right)_{1}\right) & \cdots & (-1)^{n} \operatorname{det} M\left(\left(\alpha_{1}\right)_{1},\left(\alpha_{1}\right)_{n-1}\right) \\
\vdots & \vdots & \cdots & \vdots \\
0 & (-1)^{n} \operatorname{det} M\left(\left(\alpha_{1}\right)_{1},\left(\alpha_{1}\right)_{n-1}\right) & \cdots & \operatorname{det} M\left(\left(\alpha_{1}\right)_{n-1},\left(\alpha_{1}\right)_{n-1}\right)
\end{array}\right| \text {, }
\end{aligned}
$$

And now apply an induction argument to get the result.

Numerical Example: Let $M=\left[\begin{array}{ccc}3 & -1 & 0 \\ -2 & 5 & -1 \\ 0 & -2 & 3\end{array}\right]$ be strictly diagonally dominant M-matrix, then $C_{2}(M)=\left[\begin{array}{ccc}13 & -3 & 1 \\ -6 & 9 & -3 \\ 4 & -6 & 13\end{array}\right]$ and $S\left(C_{2}(M)\right)^{\mathrm{T}} S=\left[\begin{array}{ccc}13 & 6 & 4 \\ 3 & 9 & 6 \\ 1 & 3 & 13\end{array}\right]$ is totally nonnegative.

Note that $\operatorname{det}(M)=33$ and $\operatorname{det}\left(S\left(C_{2}\right)^{\mathrm{T}} S\right)=33^{2}=1089$

Numerically we can conclude the following fact.

Fact: For any $n \times n$ tridiagonal $M$-matrix $M=\left(m_{i j}\right)$ the following formula is true.

$$
\operatorname{det} M^{-1}(1, \cdots, n-1 / 2, \cdots, n)=\operatorname{det} M^{-1}(2, \cdots, n / 1, \cdots, n-1)=0 \text { for } n \geq 3
$$

Moreover, $\operatorname{det} M^{-1}=\frac{1}{\operatorname{det} M}$

To prove this result we use Theorem 4.1.

$$
\begin{aligned}
& \text { Suppose } M \text { is nonsingular then } M^{-1}=\frac{S\left(C_{n-1}(M)\right)^{\mathrm{T}} S}{\operatorname{det} M} \text {, so } \\
& \qquad \operatorname{det} M^{-1}=\operatorname{det}\left(\frac{S\left(C_{n-1}(M)\right)^{\mathrm{T}} S}{\operatorname{det} M}\right)=\frac{\operatorname{det}\left(S\left(C_{n-1}(M)\right)^{\mathrm{T}} S\right)}{(\operatorname{det} M)^{n}}=\frac{(\operatorname{det} M)^{n-1}}{(\operatorname{det} M)^{n}}=\frac{1}{\operatorname{det} M}
\end{aligned}
$$

For example, when $n=3$, the $M$-matrix of our form 


$$
\begin{gathered}
M=\left[\begin{array}{ccc}
a_{1} & -b_{1} & 0 \\
-c_{1} & a_{2} & -b_{2} \\
0 & -c_{2} & a_{3}
\end{array}\right] \text { has an inverse given as } \\
M^{-1}=\left[\begin{array}{llll}
m_{11}^{-1} & m_{12}^{-1} & m_{12}^{-1} \\
m_{21}^{-1} & m_{22}^{-1} & m_{23}^{-1} \\
m_{31}^{-1} & m_{32}^{-1} & m_{33}^{-1}
\end{array}\right]=\left[\begin{array}{ccc}
\frac{\operatorname{det} M(2,3 / 2,3)}{\operatorname{det} M} & -\frac{\operatorname{det} M(1,3 / 2,3)}{\operatorname{det} M} & \frac{\operatorname{det} M(1,2 / 2,3)}{\operatorname{det} M} \\
-\frac{\operatorname{det} M(2,3 / 1,3)}{\operatorname{det} M} & \frac{\operatorname{det} M(1,3 / 1,3)}{\operatorname{det} M} & -\frac{\operatorname{det} M(1,2 / 1,3)}{\operatorname{det} M} \\
\operatorname{det} M(2,3 / 1,2) & -\frac{\operatorname{det} M(1,3 / 1,2)}{\operatorname{det} M} & \frac{\operatorname{det} M(1,2 / 1,2)}{\operatorname{det} M}
\end{array}\right] \\
=\left(b_{1} a_{3}\right)\left(a_{1} b_{2}\right)-\left(b_{1} b_{2}\right)\left(a_{1} a_{3}\right)=0 .
\end{gathered}
$$

Similarly we can find $\operatorname{det} M^{-1}(2,3 / 1,2)=0$.

Illustrative Example: Let $M=\left[\begin{array}{ccc}3 & -1 & 0 \\ -1 & 4 & -2 \\ 0 & -3 & 5\end{array}\right]$ be a tridiagonal $M$-matrix and

$$
M^{-1}=\left[\begin{array}{lll}
0.3784 & 0.1351 & 0.0541 \\
0.1351 & 0.4054 & 0.1622 \\
0.0811 & 0.2432 & 0.2973
\end{array}\right]
$$

Note that $\operatorname{det} M^{-1}(1,2 / 2,3)=\operatorname{det} M^{-1}(2,3 / 1,2) \cong 0$

Observe that the error came from the rounded to the nearest part of 10,000.

Theorem 4.2 Let $M$ be a strictly diagonally dominant $M$-matrix, if $\alpha=\{1, \cdots, n-1\}, \quad \beta=\{2, \cdots, n\}$, $\gamma=\alpha \bigcap \beta$ then

$$
\operatorname{det} M^{-1}=\frac{\operatorname{det} M^{-1}[\alpha \mid \alpha] \times \operatorname{det} M^{-1}[\beta \mid \beta]}{\operatorname{det} M^{-1}(\gamma)}
$$

\section{Proof:}

$$
\text { Assume } M^{-1}=\left[\begin{array}{ccc}
a & m_{12}^{-1} & b \\
m_{21}^{-1} & M_{22}^{-1} & m_{23}^{-1} \\
c & m_{32}^{-1} & d
\end{array}\right] \text { and }
$$

$$
M^{-1}[\alpha \mid \alpha]=\left[\begin{array}{cc}
a & m_{12}^{-1} \\
m_{21}^{-1} & M_{22}^{-1}
\end{array}\right], \quad M^{-1}[\alpha \mid \beta]=\left[\begin{array}{cc}
m_{12}^{-1} & b \\
M_{22}^{-1} & m_{23}^{-1}
\end{array}\right], \quad M^{-1}[\beta \mid \alpha]=\left[\begin{array}{cc}
m_{21}^{-1} & M_{22}^{-1} \\
c & m_{32}^{-1}
\end{array}\right], \quad M^{-1}[\beta \mid \beta]=\left[\begin{array}{cc}
M_{22}^{-1} & m_{23}^{-1} \\
m_{32}^{-1} & d
\end{array}\right] .
$$

Note that $M^{-1}[\alpha \mid \beta]=M^{-1}[\beta \mid \alpha]=0$ by previous fact, and by using Sylvester's identity, we have

$$
\begin{aligned}
\operatorname{det} M^{-1} & =\frac{\operatorname{det} M^{-1}[\alpha \mid \alpha] \times \operatorname{det} M^{-1}[\beta \mid \beta]-\operatorname{det} M^{-1}[\alpha \mid \beta] \times \operatorname{det} M^{-1}[\beta \mid \alpha]}{\operatorname{det} M_{22}^{-1}} \\
& =\frac{\operatorname{det} M^{-1}[\alpha \mid \alpha] \times \operatorname{det} M^{-1}[\beta \mid \beta]}{\operatorname{det} M_{22}^{-1}} .
\end{aligned}
$$

Moreover we conclude the following theorem.

Theorem 4.3 Let $M$ be the $M$-matrix defined above then 


$$
\operatorname{det}\left(M^{-1}-x E_{i i}\right)=\operatorname{det} M^{-1}-\frac{x \times a_{i}}{\operatorname{det} M}
$$

For example

$$
\begin{gathered}
M=\left[\begin{array}{cccc}
2 & -1 & 0 & 0 \\
-1 & 3 & -1 & 0 \\
0 & -2 & 4 & -1 \\
0 & 0 & -3 & 5
\end{array}\right], M^{-1}=\left[\begin{array}{llll}
0.6308 & 0.2615 & 0.0769 & 0.0154 \\
0.2615 & 0.5231 & 0.1538 & 0.0308 \\
0.1538 & 0.3077 & 0.3846 & 0.0769 \\
0.0923 & 0.1846 & 0.2308 & 0.2462
\end{array}\right] \\
\text { Let } x=0.2 \text { then } M^{-1}+0.2 E_{11}=\left[\begin{array}{llll}
0.4308 & 0.2615 & 0.0769 & 0.0154 \\
0.2615 & 0.5231 & 0.1538 & 0.0308 \\
0.1538 & 0.3077 & 0.3846 & 0.0769 \\
0.0923 & 0.1846 & 0.2308 & 0.2462
\end{array}\right] \\
\operatorname{det}\left(M^{-1}+0.2 E_{11}\right)=0.0092 \text { and } \operatorname{det} M^{-1}-\frac{x \times a_{1}}{\operatorname{det} M}=0.0154-\frac{0.2 \times 2}{65}=0.0092
\end{gathered}
$$

Now, we will perturb elements inside the diagonal band of the inverse of $M$-matrix without losing the nonnegativity property. We begin with the $(1,1)$ element then generalize to other elements.

Theorem 4.4 Let $M$ be a strictly diagonally dominant tridiagonal $M$-matrix. Then the matrix $M^{-1}-x E_{11}$ is totally nonnegative for all $x \in\left[0, \frac{1}{a_{1}}\right]$.

Proof:

$$
\text { Let } M=\left[\begin{array}{ccccc}
a_{1} & -b_{1} & 0 & \ldots & 0 \\
-c_{1} & a_{2} & -b_{2} & \ldots & 0 \\
0 & \ddots & \ddots & \ddots & \vdots \\
\vdots & \cdots & -c_{n-2} & a_{n-1} & -b_{n-1} \\
0 & \cdots & 0 & -c_{n-1} & a_{n}
\end{array}\right]
$$

Be a nonsingular strictly diagonally dominant tridiagonal $M$-matrix then $M^{-1}$ is totally nonnegative.

By Lemma 3.5 and Proposition 3.2, we have

$$
M^{-1}-x E_{11} \text { is totally nonnegative } \forall x \in\left[0, \frac{\operatorname{det} M^{-1}}{\operatorname{det} M^{-1}\{2, \cdots, n\}}\right] \text {. }
$$

By using the formula in Proposition 3.3

$$
\frac{\operatorname{det} M^{-1}}{\operatorname{det} M^{-1}\{2, \cdots, n\}}=\frac{(-1)^{1+1} \frac{\operatorname{det} M^{-1}\{2, \cdots, n\}}{a_{1}}}{\operatorname{det} M^{-1}\{2, \cdots, n\}}=\frac{1}{a_{1}} .
$$

Note that a similar result holds for decreasing the element $m_{n n}^{-1}$ by considering the matrix $\tilde{S} M^{-1} \tilde{S}$, which reverses the matrix $M^{-1}$ as the relation $m_{i j}^{-1}=m_{n-i+1, n-j+1}^{-1}$.

We can generalize this result for the other elements of diagonal.

Theorem 4.5 Assume $M$ is a strictly diagonally dominant tridiagonal $M$-matrix. Then the matrix $M^{-1}-x E_{i i}$ is totally nonnegative for all $x \in\left[0, \frac{1}{a_{i}}\right]$.

Proof: Suppose that $X=M^{-1}-x E_{i i}$ is not totally nonnegative for all $x \in\left[0, \frac{1}{a_{i}}\right]$, then there exist $\alpha, \beta \in Q_{n, k}$ both contain $i$ such that $\operatorname{det} X=\operatorname{det}\left(\left(M^{-1}-x E_{i i}\right)(\alpha, \beta)\right)<0$.

To compute $\operatorname{det} X$ expand the determinant along the $i^{\text {th }}$ row of $X$ then 
$\operatorname{det} X=(-1)^{\alpha_{i}+\beta_{i}}(-x) \operatorname{det} X\left(\left\{\alpha_{i}\right\},\left\{\beta_{i}\right\}\right)+\operatorname{det} M^{-1}\left(\alpha^{\prime}, \beta^{\prime}\right)$ where $\alpha^{\prime} \subset \alpha, \quad \beta^{\prime} \subset \beta$, and $\operatorname{det} M^{-1}\left(\alpha^{\prime}, \beta^{\prime}\right)$ is some minor of $M^{-1}$.

Take the case when $\alpha_{i}+\beta_{i}$ odd. Thus, $\operatorname{det} X$ is a positive linear compination of minors of $M^{-1}$ and hence is positive, which contradicts the assumption.

Now suppose $\exists x \notin\left[0, \frac{1}{a_{i}}\right]$ such that $M^{-1}-x E_{i i}$ is totally nonnegative matrix.

Suppose that $x>\frac{1}{a_{i}}$, then by Theorem 4.3.

$$
\operatorname{det}\left(M^{-1}+x E_{i i}\right)=\operatorname{det} M^{-1}-\frac{x \times a_{i}}{\operatorname{det} M}<\operatorname{det} M^{-1}-\frac{1}{\operatorname{det} M}=0, \text { since } \operatorname{det} M^{-1}=\frac{1}{\operatorname{det} M}
$$

which contradicts the nonnegativity of $M^{-1}-x E_{i i}$.

$$
\begin{aligned}
\text { Numerical Example: Let } M & =\left[\begin{array}{cccc}
2 & -1 & 0 & 0 \\
-1 & 3 & -1 & 0 \\
0 & -2 & 4 & -1 \\
0 & 0 & -3 & 5
\end{array}\right] \text { is strictly diagonally dominant tridiagonal } M \text {-matrix } \\
M^{-1} & =\left[\begin{array}{cccc}
0.6308 & 0.2615 & 0.0769 & 0.0154 \\
0.2615 & 0.5231 & 0.1538 & 0.0308 \\
0.1538 & 0.3077 & 0.3846 & 0.0769 \\
0.0923 & 0.1846 & 0.2308 & 0.2462
\end{array}\right] \text { so }
\end{aligned}
$$

The matrices

$$
\begin{aligned}
& M^{-1}+x E_{11}=\left[\begin{array}{cccc}
{[0.1308,0.6308]} & 0.2615 & 0.0769 & 0.0154 \\
0.2615 & 0.5231 & 0.1538 & 0.0308 \\
0.1538 & 0.3077 & 0.3846 & 0.0769 \\
0.0923 & 0.1846 & 0.2308 & 0.2462
\end{array}\right], x \in[0,0.5], \\
& M^{-1}+\chi E_{22}=\left[\begin{array}{cccc}
0.6308 & 0.2615 & 0.0769 & 0.0154 \\
0.2615 & {[0.1898,0.5231]} & 0.1538 & 0.0308 \\
0.1538 & 0.3077 & 0.3846 & 0.0769 \\
0.0923 & 0.1846 & 0.2308 & 0.2462
\end{array}\right], x \in[0,0.3333] \text {, } \\
& M^{-1}+x E_{33}=\left[\begin{array}{cccc}
0.6308 & 0.2615 & 0.0769 & 0.0154 \\
0.2615 & 0.5231 & 0.1538 & 0.0308 \\
0.1538 & 0.3077 & {[0.1346,0.3846]} & 0.0769 \\
0.0923 & 0.1846 & 0.2308 & 0.2462
\end{array}\right], x \in[0,0.25] \text {, and } \\
& M^{-1}=\left[\begin{array}{cccc}
0.6308 & 0.2615 & 0.0769 & 0.0154 \\
0.2615 & 0.5231 & 0.1538 & 0.0308 \\
0.1538 & 0.3077 & 0.3846 & 0.0769 \\
0.0923 & 0.1846 & 0.2308 & {[0.0462,0.2462]}
\end{array}\right], x \in[0,0.2]
\end{aligned}
$$

are TNN matrices.

Note that $\operatorname{det} M^{-1}(\{1,2,3\} \backslash\{2,3,4\})=\operatorname{det} M^{-1}(\{2,3,4\} \backslash\{1,2,3\})=0$.

\section{References}

[1] McDonald, J.J., Nabben, R., Neumannand, M., Schneider, H. and Tsatsomeros, M.J. (1998) Inverse Tridiagonal 
Z-Matrices. Linear and Multilinear Algebra, 45, 75-97. http://dx.doi.org/10.1080/03081089808818578

[2] Berman, A. and Plemmons, R. (1979) Nonnegative Matrices in the Mathenlatical Sciences. Academic, New York.

[3] Peña, J.M. (1995) M-Matrices Whose Inverses Are Totally Positive. Linear Algebra and Its Applications, 221, 189193. http://dx.doi.org/10.1016/0024-3795(93)00244-T

[4] Ostrowski, A. (1937) Über die Determinanten mit überwiegender Hauptdiagonale. Commentarii Mathematici Helvetici, 10, 69-96. http://dx.doi.org/10.1007/BF01214284

[5] Minkowski, H. (1900) Zur Theorie der Einheiten in den algebraischen Zahlkörper. Nachrichten von der Königl. Gesellschaft der Wissenschaften und der Georg-Augusts-Universität zu Göttingen Mathematisch-Physikalische Klasse: Fachgruppe II, Nachrichten aus der Physik, Astronomie, Geophysik, Technik, 90-93.

[6] Minkowski, H. (1907) Diophantische Approximationen. Tuebner, Leipzig. http://dx.doi.org/10.1007/978-3-663-16055-7

[7] Horn, R. and Johnson, C. (1991) Topics in Matrix Analysis. Cambridge University Press, Cambridge. http://dx.doi.org/10.1017/CBO9780511840371

[8] Adm, M. and Garloff, J. (2014) Invariance of Total Nonnegativity of a Tridiagonal Matrix under Element-Wise Perturbation. Operators and Matrices, 8, 129-137. http://dx.doi.org/10.7153/oam-08-06

[9] Ando, T. (1987) Totally Positive Matrices. Linear Algebra and Its Applications, 90, 165-219. http://dx.doi.org/10.1016/0024-3795(87)90313-2

[10] Pinkus, A. (2010) Totally Positive Matrices. Cambridge Tracts in Mathematics (No. 181). Cambridge University Press, Cambridge.

[11] Fallat, S.M. and Johnson, C.R. (2011) Totally Nonnegative Matrices. Princeton University Press, Princeton, Oxford. http://dx.doi.org/10.1515/9781400839018

[12] Adam, M. and Garloff, J. (2013) Interval of Totally Nonnegative Matrices. Linear Algebra and Its Applications, 439, 3796-3806. http://dx.doi.org/10.1016/j.laa.2013.10.021 